\title{
Brain tissue transcriptomic analysis of SIV-infected macaques identifies Poly (ADP-ribose) polymerases (PARPs) as potential biomarkers for neuropathogenesis.
}

Carla Mavian

University of Florida https://orcid.org/0000-0003-3819-5520

Andrea S Ramirez-Mata

University of Florida

James Jarad Dollar

Latvijas Universitate

David J Nolan

University of Florida

Shannan N Rich

University of Florida

Kevin White

Boston College

Brittany Rife Magalis

University of Florida

Melanie N Cash

University of Florida

Simone Marini

University of Florida

Mattia Prosperi

University of Florida

David Moraga Amador

University of Florida

Alberto Riva

University of Florida

Kenneth C Williams

Boston College

Marco Salemi ( $\square$ salemi@pathology.ufl.edu )

University of Florida https://orcid.org/0000-0003-0136-2102 
Research

Keywords: SIV, HIV, brain, transcriptomics, PARPs

Posted Date: May 28th, 2020

DOI: https://doi.org/10.21203/rs.3.rs-30629/v1

License: (c) (i) This work is licensed under a Creative Commons Attribution 4.0 International License. Read Full License 


\section{Brain tissue transcriptomic analysis of SIV-infected macaques identifies Poly (ADP-ribose) polymerases (PARPs) as potential biomarkers for neuropathogenesis.}

Carla Mavian ${ }^{1,2 *}$, Andrea S. Ramirez-Mata ${ }^{1,2 \$}$, James Jarad Dollar ${ }^{2 \$}$, David J. Nolan ${ }^{1,2 \$ \#}$, Shannan N. Rich $^{2,3}$, Kevin White ${ }^{4}$, Brittany Rife Magalis ${ }^{1,2}$, Melanie Cash ${ }^{1,2}$, Simone Marini ${ }^{2,3}$, Mattia C. F. Prosperi ${ }^{2,3}$, David Moraga Amador ${ }^{5}$, Alberto Riva ${ }^{5}$, Kenneth C. Williams ${ }^{4}$, Marco Salemi ${ }^{1,2 *}$

*= corresponding author: cmavian@ufl.edu; salemi@pathology.ufl.edu

$\$=$ These authors contributed equally to this work.

\section{Affiliations}

1 Department of Pathology, Immunology, and Laboratory Medicine, College of Medicine, University of Florida, Gainesville, FL, USA; 2 Emerging Pathogens Institute, University of Florida, Gainesville, FL, USA; 3 Department of Epidemiology, University of Florida, Gainesville, FL, USA; 4 Biology Department, Boston College, Boston, MD, USA. 5.Interdisciplinary Center for Biotechnology Research (ICBR), University of Florida, Gainesville, FL, USA; \#= present address: Bioinfoexperts, LLC, 718 Bayou Ln, Thibodaux, LA, 70301, USA

\footnotetext{
Abstract

Background. Despite improvements in antiretroviral therapy, human immunodeficiency virus type 1 (HIV1)-associated neurocognitive disorders (HAND) remain prevalent in subjects undergoing therapy. HAND significantly affects individuals' quality of life, as well as adherence to therapy, and, despite the increasing understanding of neuropathogenesis, no definitive diagnostic or prognostic marker has been identified. Results. We investigated transcriptomic profiles in frontal cortex tissues of Simian immunodeficiency virus (SIV)-infected Rhesus macaques sacrificed at different stages of infection. Gene expression was compared among SIV-infected animals $(n=11)$, with or without CD8+ lymphocyte depletion, based on detectable $(n=6)$ or non-detectable $(n=5)$ presence of the virus in frontal cortex tissues. Significant enrichment in activation of monocyte and macrophage cellular pathways was found in animals with detectable brain infection, independently from CD8+ lymphocyte depletion. In addition, transcripts of four
} 
poly (ADP-ribose) polymerases (PARPs) were up-regulated in the frontal cortex, which was confirmed by real-time polymerase chain reaction.

Conclusions. Our results shed light on involvement of PARPs in SIV infection of the brain and their role in SIV-associated neurodegenerative processes. Inhibition of PARPs may provide an effective novel therapeutic target for HIV-related neuropathology.

\section{Keywords}

SIV, HIV, brain, transcriptomics, PARPs 


\section{Background}

The advent of combination antiretroviral therapy (CART) resulted in a 50\% decline in rates of AIDS-related deaths, and a $40-50 \%$ decrease in the incidence of human immunodeficiency virus (HIV)-associated dementia (HAD) [1]. Yet an estimated 50\% of infected patients exhibit HIV-1 central nervous system (CNS) infection [2], with approximately $30 \%$ of in people living with HIV (PLWH) progressing to some form of HIVassociated neurocognitive disorder (HAND) [3]. Even in HIV-infected individuals on combined anti-retroviral therapy (cART), low-level viral replication persists in the central nervous system (CNS) [4]. Residual viremia as a result of incompletely suppressive cART $[5,6]$ is associated with low-level immune activation driving chronic inflammation [7, 8]. It has been shown that both HIV and Simian immunodeficiency virus (SIV) can enter the CNS during early stages of infection $[9,10]$, and there is compelling evidence that the brain is a putative reservoir for HIV [11, 12]. Persistent CNS infection and inflammation may contribute to the development of HAND [13], which remains a major cause of morbidity among HIV-infected individuals. As HAND-related cognitive decline is exacerbated by age-associated neurodegeneration, the prevalence of HAND is only expected to escalate with cART-increased life expectancy [14]. Moreover, if therapy is interrupted, viral rebound is going to occur [15-17], and because HIV is able to replicate in the CNS, brain specific viral variants are found at rebound after interruption of cART [18].

While progress has been made in understanding the pathophysiology of HAND and neurological complications of HIV acquired immunodeficiency syndrome (neuroAIDS) under conditions of high viral load, the host's inflammatory responses to low-level chronic systemic infection and how this exacerbates neuronal injury and dysfunction in the brain are incompletely understood. Infection of Rhesus macaques (Mucaca mulatta) with simian immunodeficiency virus (SIV) in the absence of therapy offers a wellestablished animal model for the study of the relationship of HIV infection and neuropathogenesis [10, 19, 20], while avoiding the confounding factor of cART [21-23]. Approximately $30 \%$ of Rhesus macaques infected with the heterogeneous SIVmac251 viral swarm [24] develop within 2-3 years [25, 26] SIVassociated encephalitis (SIVE), the pathological hallmark of neuroAIDS, which is diagnosed post mortem by the presence of virus and abnormal histopathology features, such as inflammation of brain tissues and formation of multinucleated giant cells. When animals are depleted of CD8+ lymphocytes using an antiCD8+ antibody before virus inoculation [27], the incidence is elevated to $>85 \%$ in less than six months. 
Thus, CD8+ lymphocyte depletion provides a useful, rapid disease model with increased incidence of brain infection and neuropathology [28, 29].

Myeloid cells accumulate in the meninges and choroid plexus during early infection, and in the perivascular space and SIVE lesions in infected macaques during late infection [30]. In particular, SIVE lesions are composed of CD68+ CD163+ macrophages during early infection, as well as SIV-infected macrophages recruited terminally during simian AIDS (SAIDS) [30, 31]. SIV-induced products of activated macrophages and astrocytes lead to CNS dysfunction and disease that might directly damage neurons [32]. These observations indicate that neuropathogenesis of HIV infection and pathogenesis of HAD and HAND may be linked [33]. It has also suggested that, given the neuroprotective properties of poly(ADPribose) polymerases (PARPs) inhibitors [34], these inhibitors might be used as neuroprotective against NeuroAIDS as well [35]. PARPs regulate a vast variety of cellular processes [36], and in particular, PARP1 and PARP-2 participate in regulating DNA metabolism [37], including DNA repair activated by DNA strand breaks [38]. Previous studies demonstrated that PARP1 plays a role of in regulating HIV replication and integration $[39,40]$.

Based on the hypothesis that PARPs appears to play an important role in HIV infection, we investigated the transcriptome of SIV-infected macaques with and without detectable virus in the brain to investigate whether PARPs expression is associated with SIV neuropathogenesis and biological processes translatable to HIV brain infection. We focused our analysis on characterizing the transcriptome profiles of the frontal cortex, as severity of cognitive impairment has been previously associated with the degree of frontal cortex neurodegeneration [41, 42]. In what follows, we report, for the first time, significant dysregulation of PARPs expression in SIV-infected brain tissues with detectable virus, associated with neurodegenerative processes. Our findings suggest that PARPs may represent new potential biomarkers, as well as therapeutic targets for HIVISIV brain infection.

\section{Methods}

\section{Animal cohorts and sample collection}

Frontal cortex tissue samples were collected from two cohorts of animals intravenously infected with SIVmac251 [24], which originally consisted of five CD8+ lymphocyte-depleted (D) and six non-CD8- 
depleted ( $\mathrm{N}$ = naturally progressing to SAIDS) Rhesus macaques, as previously described (Table S1) [43]. Procedures on the CD8+ lymphocyte-depleted and naturally progressing cohort were conducted with the approval of New England Regional Primate Center at Harvard [19] and University Tulane University's Institutional Animal Care and Use Committee [43], respectively. Animals were kept in the same facility under similar conditions to minimize batch effects. Additional information on the treatment and handling of macaques in this cohort can be found in the study of Strickland et al. [44]. Gross pathology of the naturally progressing animals can be found in Rife et al. [43], and of the CD8+ lymphocyte-depleted ones in Table S1. All tissues collected during necropsy, following SAIDS onset and humane sacrifice, with the exception of animals N06, N07, D01, and D02, which were euthanized at 21 days post-infection (DPI) [43] (Table S1), were snap frozen in optimal cutting temperature medium and stored at $-80^{\circ} \mathrm{C}$. A single $50-$ $100 \mathrm{mg}$ section of frontal cortex tissue was used for RNA isolation. Viral DNA was extracted from frontal cortex tissues and detected by single genome sequencing (SGS) of the SIV envelope gene sequence as previously described $[10,43]$.

\section{RNA isolation and Next Generation Sequencing (RNA-Seq)}

Total RNA was extracted with Qiagen RNeasy Lipid Tissue Mini Kit (Cat No: 74804) according to manufacturer protocol. Quantity and quality of RNA, from post mortem frontal cortex tissue samples, was assessed using the Invitrogen Qubit 2.0 and Agilent Tapestation 2200, respectively. Frontal cortex RNA sequencing libraries were prepared with Illumina TruSeq Stranded mRNA HT kit and sequenced on the 2x100 paired-end Illumina NextSeq platform at the University of Florida Interdisciplinary Center for Biotechnology Research.

\section{RNA-Seq data analysis}

Paired-end reads were trimmed using trimmomatic ( $v$ 0.36) [45], and quality control on the original and trimmed reads was performed using FastQC $(v$ 0.11.4) [46]. Trimmed paired-end reads were mapped to the Macaca mulatta genome available at Ensembl (http://dec2015.archive.ensembl.org/Macaca mulatta/Info/Index). Sequences were aligned with STAR (v2.6.1) [47]. Reads were submitted to the Sequence Read Archive with the BioProject PRJNA624871. 
We obtained an average of $\sim 30.5$ million reads for each sample, with an average of $55.9 \%$ of the reads mapped to the reference genome (Table S2), in line with typical percentage of transcriptome mapping [48] (Table S2). Gene expression was quantified using RSEM (v1.2.31) [49]. Differential expression analysis was performed using DESeq2 [50], using a fold-change threshold of 0.05 and a significance level $P<0.01$. Gene Ontology, Disease Association Enrichment and Pathway Analysis of DEGs were determined using the Ingenuity Pathway Analysis (IPA) software (Quiagen) after importing the list of 152 up-regulated (cut off of $\left(\log _{2}(\log 2)\right.$ Fold-Change $\left.(F C)\right)$ of 1) and five down-regulated DEGs (cut off of Log2(FC)-1), and P-value $<=0.05$. The -log(p-value) of the pathway indicated the significance of overlap of the genes observed and the ones in the pathway and is calculated using the Fisher's Exact Test [51]. Prediction of activation or de-activation of a certain pathway is based on the z-score, using a z-score threshold of 1.3. Calculation of the z-score of a pathway, which assess the match of observed and predicted up/downregulation patterns, is based on comparison between the direction of the genes observed compared to direction of those same genes in the active state of the pathway [52].

\section{Quantitative PCR (qPCR)}

cDNA from frontal cortex was generated with Invitrogen Superscript IV and random hexamers according to manufacturer's protocols, using aliquots from RNA isolated for RNA sequencing. Comparative qPCR was conducted in triplicate for each sample using Applied Biosystems TaqMan Universal PCR Master Mix (ThermoFisher Catalog number: 4304437) and probes (0.25 $\mu \mathrm{M})$ for PARP9, PARP12, PARP14, and Glyceraldehyde 3-phosphate dehydrogenase (GAPDH). Comparative qPCR was conducted with a 10minute hold at $95^{\circ} \mathrm{C}$, followed by 45 cycles of $95^{\circ} \mathrm{C}$ for 15 seconds and $60^{\circ} \mathrm{C}$ for 1 minute on the Applied Biosystems 7500 Fast Real-Time PCR System. Each sample's mean Ci value for each qPCR reaction was normalized by subtracting the sample's mean $\mathrm{C}_{\mathrm{T}}$ for GAPDH to generate $\Delta \mathrm{C}_{\mathrm{T}}$. $\mathrm{A}$ standard deviation for the qPCR reaction was normalized with the standard deviation of GAPDH: SADJUSTED $=\left(\mathrm{S}_{\mathrm{PROBE}}{ }^{2}+\right.$ $\left.\mathrm{S}_{\mathrm{GAPDH}^{2}}\right)^{1 / 2} . \Delta \Delta \mathrm{C}_{\mathrm{T}}$ was calculated for each sample by subtracting its $\Delta \mathrm{C}_{\mathrm{T}}$ value from the mean $\Delta \mathrm{CT}$ value of the samples without detectable virus in the brain. The fold difference in reference to the group of macaques without detectable virus in the brain was calculated with $2^{-\triangle \Delta C T}$ and error bars were calculated 
with $2^{-\triangle \triangle C T} \pm$ SADJUSTED. Statistical significance was tested using a Welch's $\mathrm{t}$-test, the hypothesis that two populations have equal means [53].

\section{Results}

\section{Transcriptomic profiles are independent of CD8+ lymphocytes depletion}

SGS of SIV env gp120 detected viral sequences in frontal cortex tissue of eight out of eleven animals: all five of the CD8+ lymphocyte-depleted and three of the non-depleted ones (Table S1). However, while the number of positive PCRs at end point dilution varied between seven and 24 in most animals, only one in animal D08 and two in N09 were detected, suggesting low level of brain infection, as previously shown [43]. Macaques with seven or more SIV sequences in the frontal cortex were all diagnosed with SIVE or meningitis at necropsy, with the exception of N02 (Table S1), which also displayed absence a viral compartmentalization in the brain [43].

For all animals, RNA-Seq of frontal cortex samples resulted in high coverage (Table S2). Comparison of gene expression profiles between CD8+ lymphocyte-depleted and non-depleted macaques showed only one significant difference - the nerve growth factor (NGF) gene, which resulted under-expressed in depleted animals with a fold change of $\log 2(F C)=3.3$ - indicating that animals could be grouped, for further comparisons, independently of depletion status. Analysis of transcripts normalized expression among macaques corroborated that depleted and non-depleted animals were inter-mixed (Figure 1). However, the analysis also revealed that macaques with $<3$ sequences were clustering with macaques with no detectable sequences in the brain. Therefore, in order to minimize gene expression noise within the data due to inter-animal variability, macaques' gene expression profiles were separated on the basis of a cut-off of $n>3$ SIV sequences detected by SGS in the brain (Figure 1). Based on this cut-off, two nonoverlapping groups could be defined: macaques with detectable (DV) or low/undetectable (NDV) SIV in the brain (Figure 1).

\section{Elevated antiviral gene response in macaques with detectable virus in the brain}


Differential expression analysis between DV and DNV macaque groups identified 102 up-regulated, and two down-regulated, differentially expressed genes (DEGs) in macaques with detectable virus in the brain (Table S3). One of the two down-regulated DEGs (Table S3), NPAS4 (Log2(FC)-1.2) is a synaptic plasticity-promoting gene [54] crucial for synaptic connections in excitatory and inhibitory neurons and neural circuit plasticity [55]. Among the 102 up-regulated DEGs (Table S3), EPSTI1 (Log2(FC)2.8) plays a role in ensuring M1 versus M2 macrophage differentiation [56]; SLFN13 (Log2(FC)2.3) restricts HIV replication [57]; MAMU-A (Log2(FC)1.9) and MAMU-A3 (Log2(FC)1.7), comprising the major histocompatibility complex class IA in Rhesus monkeys, are linked to disease progression during SIV infection [58] (Table S3). Evidence of antiviral response is shown by up-regulation of components of interferon response, such the type I interferon (IFN)-stimulated genes (ISGs) ISG15 (Log2(FC)4.2) [59] and ISG20 (Log2(FC)4.7) [60], as well as of DDX60 (Log2(FC)3.9), a promotor of RIG1-like receptormediated signaling [61] (Table S3). Proteasome activity, influenced by PSMB8 (Log2(FC)3.3) and PSMB9 (Log2(FC)3.0), is crucial for regulation of neurotransmission through protein turnover in neuronal synapses [62], as well as for long-term memory establishment in the CNS [63] (Table S3). PSMB8 and PSMB9 have been previously implicated in research studying SIVE-induced neuronal dysfunction [64] (Table S3). Lastly, NCF1 produces superoxide anions causing increased oxidative stress, which is linked to nervous system damage $[65,66]$, and activation of STAT1 (Log2(FC)1.9) provides further evidence of response to oxidative stress [67] (Table S3).

\section{Intensification of innate immune response in macaques with detectable virus in the brain}

Enrichment in activation of monocyte and macrophage cellular pathways $(z$-score $=2)$ was indicated by DEGs such as CD74 (Log2(FC)2.6), CD37 (Log2(FC)1.9), CSF1R (Log2(FC)1.2), and MNDA (Log2(FC)1.7) (Figure 2, Table S3). CSF1, in particular, has been associated with a positive feedback system wherein HIV infection increases CSF1 expression, followed by increased susceptibility of monocytes and macrophages to HIV replication upon exposure to CSF1 [68, 69]. Extending beyond the myeloid-mediated response, innate immunity pathways were identified as significantly differentiated: interferon signaling (z-score = 3.2), macrophage migration inhibitory factor (MIF)-regulation $(z$-score $=2)$ and Toll-like receptor $(T L R)$ signaling pathways $(z$-score $=2)($ Figure 2, Table S4, S5). In line to what 
previously reported during acute SIV infection in the brain of rhesus macaques, we found that the interferon signaling pathway was predicated to be activated $(z$-score $=3.2)$ in absence of high expression of either IFN $\alpha$ or IFN $\gamma$ genes [70] (Figure 2, Table S3). Genes that were upregulated in this pathways were: IFI35 (Log2(FC)1.0), IFI6 (Log2(FC)2.0), IFIT1 (Log2(FC)2.8), IFIT3 (Log2(FC)3.3), IRF9 (Log2(FC)1.8), ISG15 (Log2(FC)4.2), MX1 (Log2(FC)2.9), OAS1 (Log2(FC)2.5), PSMB8 (Log2(FC)3.3), STAT1, (Log2(FC)1.9) and STAT2 (Log2(FC)1.4) (Table S3, S4, S5). Our results reflect previous transcriptomic studies that showed that the frontal cortex of SIV-infected macaques at terminal stage of SIVE was characterized by upregulation of STAT1, protein induced by cortical neurons, and ISG15, protein product of infiltrating macrophages [32]. While MIF is a cytokine constitutively expressed by monocytes and macrophages in large amounts [71], it is an integral mediator of the innate immune system (TLR4), whereas TLRs initiate NF-KB and a number of other signaling pathways that broadly induce pro-inflammatory cytokines (Figure 2, Table S4). Dysregulation of reactive oxygen species processes was indicated with NCF1 (Log2(FC)3.3), encoding for a NADPH oxidase that produces superoxide anions, inflammation, and organ injury through interaction with toll-like receptors such as the DEG TLR4 (Log2(FC)1.5) [72]. Intensification of innate immune response was also indicated by several DEGs, such C1QB (Log2(FC)2.0), C1QC (Log2(FC)2.3), and C3 (Log2(FC)1.5), involved in activation of complement and coagulation cascades (z-score $=2)$ (Figure 2, Table S3, Table S4). Such complement cascades work to enhance the phagocytosis, proteolysis, inflammation, and overall magnitude of immune action [73]. Complement system cascades have been linked to HIV-induced neurodegeneration in other research studies $[74,75]$ and to endothelial damage leading to reduced integrity of the blood brain barrier [76] (Figure 2, Table S4 and S5). In line with this observation, numerous genes within the neuroinflammation signaling pathway were over-expressed, leading to prediction that this pathway may be activated $(z$-score $=3.6)$ (Figure 2, Table S4 and S5), and consequent possibility that inflammation was occurring in the frontal cortex of the SIV-infected macaques with detectable virus in the brain. The fcy receptor-mediated phagocytosis in macrophages and monocytes pathway $(z$-score $=2)$ was also predicted to be activated (Figure 2, Table S4 and S5). Fc-mediated phagocytosis has been suggested as a successful mechanism for rapid control and clearance of HIV, as well as for reservoir eradication [77]. 


\section{Upregulation of PARPs in macaques with detectable virus in the brain}

Transcripts of four PARPs were up-regulated in the SIV-infected frontal cortex: PARP9 (Log2(FC)1.8), PARP10 (Log2(FC)1.9), PARP12 (Log2(FC)1.9), and PARP14 (Log2(FC)2.7) (Figure 3a, Table S3, Table

S6). Over expression of these PARPs was also corroborated by quantitative PCR (Figure 3b). Expression of PARP1, a member of the PARPs family that has been the focus of HIV research due to their role in viral integration, replication, and transcription [39, 40, 78-82], as well the other PARPs, was not significantly over or under regulated (Table S6), as also confirmed by qPCR of mRNA transcripts (Figure 3b). PARPs are known to be activated by DNA strand breaks [83, 84], such ones occurring in HIV integration, as well as by interferon response [85]. While there are mixed reports as to whether [40, 81] or not such genes are necessary for HIV integration [86, 87], their function as a transcriptional repressor of HIV and inhibitor of cellular translation is known $[82,85]$. Upregulation of PARP9, PARP10, PARP12 and PARP14 and TNFSF10 predicts the activation of the death receptor signaling pathway $(z$-score $=2.2)$, which is associated with programmed cell death (Figure 2, Table S4 and S5). Activation of pathways associated with interferon and death receptor signaling are likely to be associated with neuronal apoptosis, similarly to what reported for infection of neurotropic West Nile virus in the brain [88].

\section{Discussion}

The CNS has gained importance as a potential reservoir during persistent HIV infections and the renewed focus of intense efforts on eradication strategies [89-91]. We have presented evidence of activation of two pathways that implicate a significant myeloid response to SIV infection in the brain of a well-established model of HIV disease progression, even in macaques euthanatized early. The result agrees with HIV and SIV entry in the CNS during early infection $[9,10]$. Presence of virus in frontal cortex was linked to upregulation of gene expression, as well as neuropathology with the exception of animal N09 [43]. It is interesting to note, however, that virus compartmentalization (distinct neurotropic subpopulation) in the brain, which has been linked to neuropathogenesis [10, 19, 20], was also absent in this animal [43]. Therefore, while virus induced dysregulation of gene expression seems to play an important role, the emergence of an SIV neurotropic sub-population may be a necessary condition for the onset of neuroAIDS, at least in the macaque model. 
Akin to previous studies [70], our findings indicate that frontal cortex of macaques with detectable SIV in the brain have significant upregulation of several genes. In particular, our results support that SIV in the frontal cortex alters transcriptional pathways associated with innate immune response, neuroinflammation, oxidative stress, and cellular death, interferon/STAT1 pathway, and monocyte/macrophage migration in line with previous studies [32, 92-95]. Over expression of a high number of genes may be due to inflammation and activation of several transcription factors, signaling molecules, and interferon-associated genes, and by presence of virus in the brain. As also shown previously in macaques with acute SIV infection [70], increased interferon, innate immunity pathways, and other antiviral responses mediated by macrophages indicated general signs of infection in the brain. For the first time and differently to what previously reported [70], however, we found over expression of PARPs, which regulate different aspects of cell metabolism [36] during SIV infection. PARP9 and PARP14 cross-regulate macrophage activation [96], while PARP10 and PARP12 are interferon induced genes [85], and have shown antiviral activities such decreasing replication of avian influenza virus [97] and Zika virus [98], respectively. Overall, PARPs' activity relationship with host and virus is quite complex, and both pro and antiviral responses have been reported [99]. PARP1-mediated cascade of progression to neurodegeneration and neuroinflammation has been shown in Parkinson's and Alzheimer's disease [100]. Yet, PARP1 resulted neither over or under expressed in animals with SIV infection in the frontal cortex, suggesting that its contribution to neuroAIDS may not be significant, despite its known role in HIV suppression by regulating HIV infection and integration $[39,40]$. On the other hand, four of the 18 PARP genes - PARP9, PARP10, PARP12, PARP14 - were clearly upregulated, suggesting that inflammation may be a byproduct of PARPs activity. Excessive activation of PARPs may cause cell death [101], followed by release of cellular components into the CNS, amplification of the immune response, and eventually neurodegeneration.

In summary, we found evidence that PARPs dysregulation could provide new, key indicators of SIV brain infection and neuropathogenesis. Moreover, since PARP inhibitors have shown promising neuroprotective properties [35], similar inhibitors may be employed against HIV-related toxicity and inflammation in the brain. Additional statistical studies using a larger number of animals and in vitro experiments are needed to determine what is the role of each PARP, and which proteins within PARP- 
mediated pathways may offer promising candidates as HAND biomarkers or novel therapeutic targets. Nevertheless, our study provides novel insights that may inform drug screening and development efforts aimed at identifying specific antiviral therapies and a new class of potential therapeutic candidates for HAND.

\section{Conclusions.}

Our study indicates that PARPs are over-expressed during SIV infection of the brain. PARPs may role in SIV-associated neurodegenerative processes. Inhibition of PARPs may provide an effective novel therapeutic target for HIV-related neuropathology.

\section{List of abbreviations}

CART : combination antiretroviral therapy

HIV : human immunodeficiency virus type 1

HAND : human immunodeficiency virus type 1- associated neurocognitive disorders

SIV : Simian immunodeficiency virus

CNS : central nervous system

neuroAIDS : neurological complications of HIV acquired immunodeficiency syndrome

SIVE : SIV-associated encephalitis

SAIDS : simian AIDS

PARPs : poly(ADP-ribose) polymerases

SGS : single genome sequencing

RNA : ribonucleic acid

mRNA : messenger RNA

qPCR : quantitative polymerase chain reaction

GAPDH : Glyceraldehyde 3-phosphate dehydrogenase

DV : detectable SIV in the brain

NDV : low/undetectable SIV in the brain

DEGs : differentially expressed genes 
NPAS: Neuronal PAS Domain Protein

EPSTI : epithelial stromal interaction

SLFN : Schlafen Family Member

MAMU-A : major histocompatibility complex, class I, A (Rhesus monkey)

IFN : interferon

ISGs : type I interferon-stimulated genes

DDX : DExD/H-Box Helicase

RIG1 : retinoic acid-inducible gene I

PSMB : Proteasome 20S Subunit Beta

NCF : Neutrophil Cytosolic Factor

STAT : Signal Transducer And Activator Of Transcription

CSF1 : Colony Stimulating Factor 1

CSF1R : Colony Stimulating Factor 1 receptor

MNDA : myeloid cell nuclear differentiation antigen

MIF : Macrophage migration inhibitory factor

IFI : Interferon Induced Protein

IRF : Interferon Regulatory Factor

MX1 : MX Dynamin Like GTPase 1

OAS1 : 2'-5'-Oligoadenylate Synthetase 1

TLR : toll-like receptor

NADPH : Reduced nicotinamide adenine dinucleotide phosphate

$\mathrm{C} 1 \mathrm{Q}$ : complement component $1 \mathrm{q}$

C3: complement component 3

TNFSF10 : TNF Superfamily Member 10 


\section{Declarations}

\section{Ethics approval and consent to participate}

Procedures on the CD8+ lymphocyte-depleted and naturally progressing cohort were conducted with the approval of New England Regional Primate Center at Harvard [19] and University Tulane University's Institutional Animal Care and Use Committee [43], respectively.

\section{Consent to publish}

Not applicable

\section{Availability of data and materials}

The datasets generated during and/or analyzed during the current study are available in the Sequence Read Archive (SRA) with the BioProject PRJNA624871 and will be released by SRA after publication, https://www.ncbi.nlm.nih.gov/sra

\section{Competing interests}

The authors declare that they have no competing interests

\section{Funding}

This work was supported by NIH award R01 NS063897. MS is supported in part by the Stephany W. Holloway University Chair in AIDS Research.

\section{Authors' Contributions}

$\mathrm{CM}$ design of the work, analysis, interpretation of data, wrote the manuscript; ASRM analysis, acquisition of data; JJD analysis, acquisition of data; DJN design of the work, analysis; SNR analysis; KW acquisition of data; BRM substantively revised the manuscript; MC analysis; SM interpretation of data, revised the manuscript; MCF interpretation of data, revised the manuscript; DMA design of the work; AR creation of pipeline used in the work; KCW interpretation of data, substantively revised the manuscript; MS design of 
the work, interpretation of data, substantively revised the manuscript. All authors read and approved the final manuscript.

\section{Acknowledgements}

Not applicable 


\section{References:}

1. Maschke M, Kastrup O, Esser S, Ross B, Hengge U, Hufnagel A: Incidence and prevalence of neurological disorders associated with HIV since the introduction of highly active antiretroviral therapy (HAART). J Neurol Neurosurg Psychiatry 2000, 69:376-380.PMC1737101.10.1136/jnnp.69.3.376

2. Zhao L, Galligan DC, Lamers SL, Yu S, Shagrun L, Salemi M, McGrath MS: High level HIV-1 DNA concentrations in brain tissues differentiate patients with post-HAART AIDS dementia complex or cardiovascular disease from those with AIDS. Sci China C Life Sci 2009, 52:651-65610.1007/s11427-009-0085-5

3. Heaton RK, Clifford DB, Franklin DR, Jr., Woods SP, Ake C, Vaida F, Ellis RJ, Letendre SL, Marcotte TD, Atkinson JH, et al: HIV-associated neurocognitive disorders persist in the era of potent antiretroviral therapy: CHARTER Study. Neurology 2010, 75:2087-2096.PMC2995535.10.1212/WNL.0b013e318200d727

4. Spudich SS: Immune activation in the central nervous system throughout the course of HIV infection. Curr Opin HIV AIDS 2016, 11:226233.PMC4773903.10.1097/COH.0000000000000243

5. Massanella M, Esteve A, Buzon MJ, Llibre JM, Puertas MC, Gatell JM, Domingo P, Stevenson M, Clotet B, Martinez-Picado J, et al: Dynamics of CD8 T-cell activation after discontinuation of HIV treatment intensification. J Acquir Immune Defic Syndr 2013, 63:152-16010.1097/QAI.0b013e318289439a

6. Massanella M, Negredo E, Puig J, Puertas MC, Buzon MJ, Perez-Alvarez N, Carrillo J, Clotet B, Martinez-Picado J, Blanco J: Raltegravir intensification shows differing effects on CD8 and CD4 T cells in HIV-infected HAART-suppressed individuals with poor CD4 T-cell recovery. AIDS 2012, 26:2285229310.1097/QAD.0b013e328359f20f

7. Massanella M, Fromentin $\mathrm{R}$, Chomont $\mathrm{N}$ : Residual inflammation and viral reservoirs: alliance against an HIV cure. Curr Opin HIV AIDS 2016, 11:234241.PMC4743501.10.1097/COH.0000000000000230

8. Klatt NR, Chomont N, Douek DC, Deeks SG: Immune activation and HIV persistence: implications for curative approaches to HIV infection. Immunol Rev 2013, 254:326-342.PMC3694608.10.1111/imr.12065

9. Resnick L, Berger JR, Shapshak P, Tourtellotte WW: Early penetration of the bloodbrain-barrier by HIV. Neurology 1988, 38:9-1410.1212/wnl.38.1.9

10. Strickland SL, Rife BD, Lamers SL, Nolan DJ, Veras NM, Prosperi MC, Burdo TH, Autissier P, Nowlin B, Goodenow MM, et al: Spatiotemporal dynamics of simian immunodeficiency virus brain infection in CD8+ lymphocyte-depleted rhesus macaques with neuroAIDS. $J$ Gen Virol 2014, 95:27842795.PMC4233634.10.1099/vir.0.070318-0

11. Marban C, Forouzanfar F, Ait-Ammar A, Fahmi F, El Mekdad H, Daouad F, Rohr O, Schwartz C: Targeting the Brain Reservoirs: Toward an HIV Cure. Front Immunol 2016, 7:397.PMC5044677.10.3389/fimmu.2016.00397

12. Wallet C, De Rovere M, Van Assche J, Daouad F, De Wit S, Gautier V, Mallon PWG, Marcello A, Van Lint C, Rohr O, Schwartz C: Microglial Cells: The Main HIV-1 Reservoir in the Brain. Front Cell Infect Microbiol 2019, 9:362.PMC6821723.10.3389/fcimb.2019.00362 
13. Valcour V, Chalermchai T, Sailasuta N, Marovich M, Lerdlum S, Suttichom D, Suwanwela NC, Jagodzinski L, Michael N, Spudich S, et al: Central nervous system viral invasion and inflammation during acute HIV infection. J Infect Dis 2012, 206:275-282.PMC3490695.10.1093/infdis/jis326

14. Fogel GB, Lamers SL, Levine AJ, Valdes-Sueiras M, McGrath MS, Shapshak P, Singer EJ: Factors related to HIV-associated neurocognitive impairment differ with age. $J$ Neurovirol 2015, 21:56-65.4320020.10.1007/s13365-014-0296-9

15. Andrade VM, Mavian C, Babic D, Cordeiro T, Sharkey M, Barrios L, Brander C, Martinez-Picado J, Dalmau J, Llano A, et al: A minor population of macrophagetropic HIV-1 variants is identified in recrudescing viremia following analytic treatment interruption. Proceedings of the National Academy of Sciences 2020:20191703410.1073/pnas.1917034117

16. Palmisano L, Giuliano M, Bucciardini R, Fragola V, Andreotti M, Galluzzo C, Pirillo MF, Weimer LE, Arcieri R, Germinario EA, et al: Determinants of virologic and immunologic outcomes in chronically HIV-infected subjects undergoing repeated treatment interruptions: the Istituto Superiore di Sanita-Pulsed Antiretroviral Therapy (ISS-PART) study. J Acquir Immune Defic Syndr 2007, 46:39-47

17. Saez-Cirion A, Bacchus C, Hocqueloux L, Avettand-Fenoel V, Girault I, Lecuroux C, Potard V, Versmisse P, Melard A, Prazuck T, et al: Post-treatment HIV-1 controllers with a long-term virological remission after the interruption of early initiated antiretroviral therapy ANRS VISCONTI Study. PLoS Pathog 2013, 9:e1003211.PMC3597518.10.1371/journal.ppat.1003211

18. Gianella S, Kosakovsky Pond SL, Oliveira MF, Scheffler K, Strain MC, De la Torre A, Letendre S, Smith DM, Ellis RJ: Compartmentalized HIV rebound in the central nervous system after interruption of antiretroviral therapy. Virus Evol 2016, 2:vew020.PMC5072458.10.1093/ve/vew020

19. Lamers SL, Nolan DJ, Rife BD, Fogel GB, McGrath MS, Burdo TH, Autissier P, Williams KC, Goodenow MM, Salemi M: Tracking the Emergence of Host-Specific Simian Immunodeficiency Virus env and nef Populations Reveals nef Early Adaptation and Convergent Evolution in Brain of Naturally Progressing Rhesus Macaques. J Virol 2015, 89:8484-8496.PMC4524235.10.1128/JVI.01010-15

20. Mallard J, Williams KC: Animal models of HIV-associated disease of the central nervous system. Handb Clin Neurol 2018, 152:41-5310.1016/B978-0-444-638496.00004-9

21. Hatziioannou T, Evans DT: Animal models for HIV/AIDS research. Nat Rev Microbiol 2012, 10:852-867.4334372.10.1038/nrmicro2911

22. Williams R, Bokhari S, Silverstein P, Pinson D, Kumar A, Buch S: Nonhuman primate models of NeuroAIDS. J Neurovirol 2008, 14:292-

$300.2715277 .10 .1080 / 13550280802074539$

23. Murray EA, Rausch DM, Lendvay J, Sharer LR, Eiden LE: Cognitive and motor impairments associated with SIV infection in rhesus monkeys. Science 1992, 255:1246-1249

24. Strickland SL, Gray RR, Lamers SL, Burdo TH, Huenink E, Nolan DJ, Nowlin B, Alvarez X, Midkiff CC, Goodenow MM, et al: Significant genetic heterogeneity of the SIVmac251 viral swarm derived from different sources. AIDS Res Hum Retroviruses 2011, 27:1327-1332.PMC3227245.10.1089/aid.2011.0100 
25. Budka H: Neuropathology of human immunodeficiency virus infection. Brain Pathol 1991, 1:163-17510.1111/j.1750-3639.1991.tb00656.x

26. Wiley CA, Achim CL, Christopherson C, Kidane Y, Kwok S, Masliah E, Mellors J, Radhakrishnan L, Wang G, Soontornniyomkij V: HIV mediates a productive infection of the brain. AIDS 1999, 13:2055-205910.1097/00002030-199910220-00007

27. Cartwright EK, Spicer L, Smith SA, Lee D, Fast R, Paganini S, Lawson BO, Nega M, Easley K, Schmitz JE, et al: CD8(+) Lymphocytes Are Required for Maintaining Viral Suppression in SIV-Infected Macaques Treated with Short-Term Antiretroviral Therapy. Immunity 2016, 45:656668.PMC5087330.10.1016/j.immuni.2016.08.018

28. Schmitz JE, Kuroda MJ, Santra S, Sasseville VG, Simon MA, Lifton MA, Racz P, Tenner-Racz K, Dalesandro M, Scallon BJ, et al: Control of viremia in simian immunodeficiency virus infection by CD8+ lymphocytes. Science 1999, 283:857-860

29. Williams K, Westmoreland S, Greco J, Ratai E, Lentz M, Kim WK, Fuller RA, Kim JP, Autissier P, Sehgal PK, et al: Magnetic resonance spectroscopy reveals that activated monocytes contribute to neuronal injury in SIV neuroAIDS. $J$ Clin Invest 2005, 115:2534-2545.1187930.10.1172/jci22953

30. Nowlin BT, Burdo TH, Midkiff CC, Salemi M, Alvarez X, Williams KC: SIV encephalitis lesions are composed of $\mathrm{CD163(+)}$ macrophages present in the central nervous system during early SIV infection and SIV-positive macrophages recruited terminally with AIDS. Am J Pathol 2015, 185:1649-

1665.PMC4450314.10.1016/j.ajpath.2015.01.033

31. Campbell JH, Hearps AC, Martin GE, Williams KC, Crowe SM: The importance of monocytes and macrophages in HIV pathogenesis, treatment, and cure. AIDS 2014, 28:2175-218710.1097/QAD.0000000000000408

32. Roberts ES, Zandonatti MA, Watry DD, Madden LJ, Henriksen SJ, Taffe MA, Fox HS: Induction of Pathogenic Sets of Genes in Macrophages and Neurons in NeuroAIDS. Am J Pathol 2003, 162:2041-2057

33. Kaul M, Zheng J, Okamoto S, Gendelman HE, Lipton SA: HIV-1 infection and AIDS: consequences for the central nervous system. Cell Death \& Differentiation 2005, 12:878-89210.1038/sj.cdd.4401623

34. Szabo C, Pacher P, Swanson RA: Novel modulators of poly(ADP-ribose) polymerase. Trends Pharmacol Sci 2006, 27:626-630.PMC2228253.10.1016/j.tips.2006.10.003

35. Rumbaugh JA, Steiner J, Sacktor N, Nath A: Developing neuroprotective strategies for treatment of HIV-associated neurocognitive dysfunction. Futur HIV Ther 2008, 2:271-280

36. Bai P: Biology of Poly(ADP-Ribose) Polymerases: The Factotums of Cell Maintenance. Mol Cell 2015, 58:947-95810.1016/j.molcel.2015.01.034

37. Ame JC, Spenlehauer C, de Murcia G: The PARP superfamily. Bioessays 2004, 26:882$89310.1002 /$ bies.20085

38. Morales J, Li L, Fattah FJ, Dong Y, Bey EA, Patel M, Gao J, Boothman DA: Review of poly (ADP-ribose) polymerase (PARP) mechanisms of action and rationale for targeting in cancer and other diseases. Crit Rev Eukaryot Gene Expr 2014, 24:1528.PMC4806654.10.1615/critreveukaryotgeneexpr.2013006875

39. Kameoka M, Nukuzuma S, Itaya A, Tanaka Y, Ota K, Ikuta K, Yoshihara K: RNA Interference Directed against Poly(ADP-Ribose) Polymerase 1 Efficiently 
Suppresses Human Immunodeficiency Virus Type 1 Replication in Human Cells. $J$ Virol 2004, 78:8931-8934

40. Ha HC, Juluri K, Zhou Y, Leung S, Hermankova M, Snyder SH: Poly(ADP-ribose) polymerase-1 is required for efficient HIV-1 integration. Proc Natl Acad Sci U S A 2001, 98:3364-3368.30659.10.1073/pnas.051633498

41. Moore DJ, Masliah E, Rippeth JD, Gonzalez R, Carey CL, Cherner M, Ellis RJ, Achim CL, Marcotte TD, Heaton RK, Grant I: Cortical and subcortical neurodegeneration is associated with HIV neurocognitive impairment. AIDS 2006, 20:879-

88710.1097/01.aids.0000218552.69834.00

42. Woods SP, Moore DJ, Weber E, Grant I: Cognitive Neuropsychology of HIVAssociated Neurocognitive Disorders. Neuropsychol Rev 2009, 19:152-168

43. Rife BD, Nolan DJ, Lamers SL, Autissier P, Burdo T, Williams KC, Salemi M: Evolution of Neuroadaptation in the Periphery and Purifying Selection in the Brain Contribute to Compartmentalization of Simian Immunodeficiency Virus (SIV) in the Brains of Rhesus Macaques with SIV-Associated Encephalitis. J Virol 2016, 90:6112-6126.PMC4907235.10.1128/JVI.00137-16

44. Strickland SL, Gray RR, Lamers SL, Burdo TH, Huenink E, Nolan DJ, Nowlin B, Alvarez X, Midkiff CC, Goodenow MM, et al: Efficient transmission and persistence of low-frequency SIVmac251 variants in CD8-depleted rhesus macaques with different neuropathology. $J$ Gen Virol 2012, 93:925-

938.PMC3541805.10.1099/vir.0.039586-0

45. Bolger AM, Lohse M, Usadel B: Trimmomatic: a flexible trimmer for Illumina sequence data. Bioinformatics 2014, 30:2114-

2120.PMC4103590.10.1093/bioinformatics/btu170

46. Brown J, Pirrung M, McCue LA: FQC Dashboard: integrates FastQC results into a web-based, interactive, and extensible FASTQ quality control tool. Bioinformatics 2017.PMC5870778.10.1093/bioinformatics/btx373

47. Dobin A, Davis CA, Schlesinger F, Drenkow J, Zaleski C, Jha S, Batut P, Chaisson M, Gingeras TR: STAR: ultrafast universal RNA-seq aligner. Bioinformatics 2013, 29:15-21.PMC3530905.10.1093/bioinformatics/bts635

48. Conesa A, Madrigal P, Tarazona S, Gomez-Cabrero D, Cervera A, McPherson A, Szcześniak MW, Gaffney DJ, Elo LL, Zhang X, Mortazavi A: A survey of best practices for RNA-seq data analysis. Genome Biology 2016, 17:1310.1186/s13059016-0881-8

49. Li B, Dewey CN: RSEM: accurate transcript quantification from RNA-Seq data with or without a reference genome. BMC Bioinformatics 2011, 12:323.PMC3163565.10.1186/1471-2105-12-323

50. Love MI, Huber W, Anders S: Moderated estimation of fold change and dispersion for RNA-seq data with DESeq2. Genome Biol 2014, 15:550.PMC4302049.10.1186/s13059-014-0550-8

51. Fisher RA: Statistical methods for research workers. Edinburgh: Oliver and Boyd; 1934.

52. Kramer A, Green J, Pollard J, Jr., Tugendreich S: Causal analysis approaches in Ingenuity Pathway Analysis. Bioinformatics 2014, 30:523530.PMC3928520.10.1093/bioinformatics/btt703

53. Welch BL: The Generalization of Students Problem When Several Different Population Variances Are Involved. Biometrika 1947, 34:28-35Doi 10.2307/2332510 
54. Margineanu MB, Mahmood H, Fiumelli H, Magistretti PJ: L-Lactate Regulates the Expression of Synaptic Plasticity and Neuroprotection Genes in Cortical Neurons: A Transcriptome Analysis. Front Mol Neurosci 2018, 11:375.PMC6191511.10.3389/fnmol.2018.00375

55. Ramamoorthi K, Fropf R, Belfort GM, Fitzmaurice HL, McKinney RM, Neve RL, Otto $\mathrm{T}$, Lin Y: Npas4 regulates a transcriptional program in CA3 required for contextual memory formation. Science 2011, 334:16691675.PMC4038289.10.1126/science. 1208049

56. Kim YH, Lee JR, Hahn MJ: Regulation of inflammatory gene expression in macrophages by epithelial-stromal interaction 1 (Epsti1). Biochem Biophys Res Commun 2018, 496:778-78310.1016/j.bbrc.2017.12.014

57. Yang JY, Deng XY, Li YS, Ma XC, Feng JX, Yu B, Chen Y, Luo YL, Wang X, Chen ML, et al: Structure of Schlafen13 reveals a new class of tRNA/rRNA- targeting RNase engaged in translational control. Nat Commun 2018, 9:1165.PMC5862951.10.1038/s41467-018-03544-X

58. Zhang ZQ, Fu TM, Casimiro DR, Davies ME, Liang X, Schleif WA, Handt L, Tussey L, Chen M, Tang A, et al: Mamu-A*01 allele-mediated attenuation of disease progression in simian-human immunodeficiency virus infection. $J$ Virol 2002, 76:12845-12854.PMC136722.10.1128/jvi.76.24.12845-12854.2002

59. Jeon YJ, Yoo HM, Chung CH: ISG15 and immune diseases. Biochim Biophys Acta 2010, 1802:485-49610.1016/j.bbadis.2010.02.006

60. Weiss CM, Trobaugh DW, Sun C, Lucas TM, Diamond MS, Ryman KD, Klimstra WB: The Interferon-Induced Exonuclease ISG20 Exerts Antiviral Activity through Upregulation of Type I Interferon Response Proteins. mSphere 2018, 3.PMC6147134.10.1128/mSphere.00209-18

61. Miyashita M, Oshiumi H, Matsumoto M, Seya T: DDX60, a DEXD/H box helicase, is a novel antiviral factor promoting RIG-I-like receptor-mediated signaling. Mol Cell Biol 2011, 31:3802-3819.PMC3165724.10.1128/MCB.01368-10

62. Speese SD, Trotta N, Rodesch CK, Aravamudan B, Broadie K: The ubiquitin proteasome system acutely regulates presynaptic protein turnover and synaptic efficacy. Curr Biol 2003, 13:899-910

63. Lopez-Salon M, Alonso M, Vianna MR, Viola H, Mello e Souza T, Izquierdo I, Pasquini $\mathrm{JM}$, Medina $\mathrm{JH}$ : The ubiquitin-proteasome cascade is required for mammalian longterm memory formation. Eur $J$ Neurosci 2001, 14:1820-1826

64. Gersten M, Alirezaei M, Marcondes MCG, Flynn C, Ravasi T, Ideker T, Fox HS: An integrated systems analysis implicates EGR1 downregulation in SIVE- induced neural dysfunction. $J$ Neurosci 2009, 29:12467-12476

65. Uzasci L, Nath A, Cotter R: Oxidative stress and the HIV-infected brain proteome. $J$ Neuroimmune Pharmacol 2013, 8:1167-1180.3714334.10.1007/s11481-013-9444-x

66. Starkov AA, Chinopoulos C, Fiskum G: Mitochondrial calcium and oxidative stress as mediators of ischemic brain injury. Cell Calcium 2004, 36:257-

26410.1016/j.ceca.2004.02.012

67. Olagnier D, Peri S, Steel C, van Montfoort N, Chiang C, Beljanski V, Slifker M, He Z, Nichols $\mathrm{CN}$, Lin R, et al: Cellular oxidative stress response controls the antiviral and apoptotic programs in dengue virus-infected dendritic cells. PLoS Pathog 2014, 10:e1004566.4270780.10.1371/journal.ppat. 1004566 
68. Haine V, Fischer-Smith T, Rappaport J: Macrophage colony-stimulating factor in the pathogenesis of HIV infection: potential target for therapeutic intervention. $J$ Neuroimmune Pharmacol 2006, 1:32-4010.1007/s11481-005-9003-1

69. Rappaport J, Volsky DJ: Role of the Macrophage in HIV-Associated Neurocognitive Disorders and Other Comorbidities in Patients on Effective Antiretroviral Treatment. J Neurovirol 2015, 21:235-241

70. Roberts ES, Burudi EM, Flynn C, Madden LJ, Roinick KL, Watry DD, Zandonatti MA, Taffe MA, Fox HS: Acute SIV infection of the brain leads to upregulation of IL6 and interferon-regulated genes: expression patterns throughout disease progression and impact on neuroAIDS. J Neuroimmunol 2004, 157:81-

9210.1016/j.jneuroim.2004.08.030

71. Calandra T, Roger T: Macrophage migration inhibitory factor: a regulator of innate immunity. Nature Reviews Immunology 2003, 3:791-80010.1038/nri1200

72. Gill R, Tsung A, Billiar T: Linking oxidative stress to inflammation: Toll-like receptors. Free Radic Biol Med 2010, 48:11211132.3423196.10.1016/j.freeradbiomed.2010.01.006

73. Janeway CA Jr TP, Walport M, et al. : The complement system and innate immunity. In Immunobiology: The Immune System in Health and Disease, 5th edition edition. New York: Garland Science; 2001.

74. Speth C, Stockl G, Mohsenipour I, Wurzner R, Stoiber H, Lass-Florl C, Dierich MP: Human immunodeficiency virus type 1 induces expression of complement factors in human astrocytes. $J$ Virol 2001, 75:2604-2615.115884.10.1128/jvi.75.6.26042516.2001

75. Bruder C, Hagleitner M, Darlington G, Mohsenipour I, Wurzner R, Hollmuller I, Stoiber H, Lass-Florl C, Dierich MP, Speth C: HIV-1 induces complement factor C3 synthesis in astrocytes and neurons by modulation of promoter activity. Mol Immunol 2004, 40:949-961

76. Orsini F, De Blasio D, Zangari R, Zanier ER, De Simoni MG: Versatility of the complement system in neuroinflammation, neurodegeneration and brain homeostasis. Front Cell Neurosci 2014, 8

77. Sips M, Krykbaeva M, Diefenbach TJ, Ghebremichael M, Bowman BA, Dugast AS, Boesch AW, Streeck H, Kwon DS, Ackerman ME, et al: Fc receptor-mediated phagocytosis in tissues as a potent mechanism for preventive and therapeutic HIV vaccine strategies. Mucosal Immunology 2016, 9:1584-159510.1038/mi.2016.12

78. Hassa PO, Hottiger MO: A role of poly (ADP-ribose) polymerase in NF-kappaB transcriptional activation. Biol Chem 1999, 380:953-95910.1515/bc.1999.118

79. Ha HC, Snyder SH: Poly(ADP-ribose) polymerase is a mediator of necrotic cell death. Proc Natl Acad Sci U S A 1999, 96:13978-13982

80. Rom S, Reichenbach NL, Dykstra H, Persidsky Y: The dual action of poly(ADPribose) polymerase -1 (PARP-1) inhibition in HIV-1 infection: HIV-1 LTR inhibition and diminution in Rho GTPase activity. Front Microbiol 2015, 6:878.4548080.10.3389/fmicb.2015.00878

81. Kameoka M, Nukuzuma S, Itaya A, Tanaka Y, Ota K, Inada Y, Ikuta K, Yoshihara K: Poly(ADP-ribose)polymerase-1 is required for integration of the human immunodeficiency virus type 1 genome near centromeric alphoid DNA in human 
and murine cells. Biochem Biophys Res Commun 2005, 334:412-

41710.1016/j.bbrc.2005.06.104

82. Bueno MT, Reyes D, Valdes L, Saheba A, Urias E, Mendoza C, Fregoso OI, Llano M: Poly(ADP-ribose) polymerase 1 promotes transcriptional repression of integrated retroviruses. $J$ Virol 2013, 87:2496-2507.3571415.10.1128/jvi.01668-12

83. Ray Chaudhuri A, Nussenzweig A: The multifaceted roles of PARP1 in DNA repair and chromatin remodelling. Nat Rev Mol Cell Biol 2017, 18:610621.PMC6591728.10.1038/nrm.2017.53

84. Ikejima M, Noguchi S, Yamashita R, Ogura T, Sugimura T, Gill DM, Miwa M: The zinc fingers of human poly(ADP-ribose) polymerase are differentially required for the recognition of DNA breaks and nicks and the consequent enzyme activation. Other structures recognize intact DNA. J Biol Chem 1990, 265:21907-21913

85. Atasheva S, Frolova EI, Frolov I: Interferon-stimulated poly(ADP-Ribose) polymerases are potent inhibitors of cellular translation and virus replication. $J$ Virol 2014, 88:2116-2130.3911523.10.1128/jvi.03443-13

86. Baekelandt V, Claeys A, Cherepanov P, De Clercq E, De Strooper B, Nuttin B, Debyser $\mathrm{Z}$ : DNA-Dependent protein kinase is not required for efficient lentivirus integration. $J$ Virol 2000, 74:11278-11285.113232.

87. Ariumi Y, Turelli P, Masutani M, Trono D: DNA damage sensors ATM, ATR, DNAPKes, and PARP-1 are dispensable for human immunodeficiency virus type 1 integration. $J$ Virol 2005, 79:2973-2978.548471.10.1128/jvi.79.5.2973-2978.2005

88. Clarke P, Leser JS, Quick ED, Dionne KR, Beckham JD, Tyler KL: Death receptormediated apoptotic signaling is activated in the brain following infection with West Nile virus in the absence of a peripheral immune response. $J$ Virol 2014, 88:10801089.PMC3911655.10.1128/JVI.02944-13

89. Saylor D, Dickens AM, Sacktor N, Haughey N, Slusher B, Pletnikov M, Mankowski JL, Brown A, Volsky DJ, McArthur JC: HIV-associated neurocognitive disorder-pathogenesis and prospects for treatment. Nat Rev Neurol 2016, 12:234248.PMC4937456.10.1038/nrneurol.2016.27

90. Hellmuth J, Valcour V, Spudich S: CNS reservoirs for HIV: implications for eradication. J Virus Erad 2015, 1:67-71.PMC4586130.

91. Salemi M, Rife B: Phylogenetics and Phyloanatomy of HIV/SIV Intra-Host Compartments and Reservoirs: The Key Role of the Central Nervous System. Curr HIV Res 2016, 14:110-120

92. Winkler JM, Chaudhuri AD, Fox HS: Translating the Brain Transcriptome in NeuroAIDS: From Non-human Primates to Humans. J Neuroimmune Pharmacol 2012, 7:372-379

93. Roberts ES, Burudi Em Fau - Flynn C, Flynn C Fau - Madden LJ, Madden Lj Fau Roinick KL, Roinick K1 Fau - Watry DD, Watry Dd Fau - Zandonatti MA, Zandonatti Ma Fau - Taffe MA, Taffe Ma Fau - Fox HS, Fox HS: Acute SIV infection of the brain leads to upregulation of IL6 and interferon-regulated genes: expression patterns throughout disease progression and impact on neuroAIDS.

94. Roberts ES, Huitron-Resendiz S, Taffe MA, Marcondes MC, Flynn CT, Lanigan CM, Hammond JA, Head SR, Henriksen SJ, Fox HS: Host response and dysfunction in the CNS during chronic simian immunodeficiency virus infection. $J$ Neurosci 2006 , 26:4577-458510.1523/jneurosci.4504-05.2006 
95. Gersten M, Alirezaei M, Marcondes MC, Flynn C, Ravasi T, Ideker T, Fox HS: An integrated systems analysis implicates EGR1 downregulation in simian immunodeficiency virus encephalitis-induced neural dysfunction. J Neurosci 2009 , 29:12467-12476.PMC2802851.10.1523/JNEUROSCI.3180-09.2009

96. Iwata H, Goettsch C, Sharma A, Ricchiuto P, Goh WW, Halu A, Yamada I, Yoshida H, Hara T, Wei M, et al: PARP9 and PARP14 cross-regulate macrophage activation via STAT1 ADP-ribosylation. Nat Commun 2016, 7:12849.PMC5095532.10.1038/ncomms 12849

97. Yu M, Zhang C, Yang Y, Yang Z, Zhao L, Xu L, Wang R, Zhou X, Huang P: The interaction between the PARP10 protein and the NS1 protein of H5N1 AIV and its effect on virus replication. Virol J 2011, 8:546.PMC3287249.10.1186/1743-422X-8546

98. Li L, Zhao H, Liu P, Li C, Quanquin N, Ji X, Sun N, Du P, Qin CF, Lu N, Cheng G: PARP12 suppresses Zika virus infection through PARP-dependent degradation of NS1 and NS3 viral proteins. Sci Signal 2018, 11.PMC6434931.10.1126/scisignal.aas9332

99. Kuny CV, Sullivan CS: Virus-Host Interactions and the ARTD/PARP Family of Enzymes. PLoS Pathog 2016, 12:e1005453.PMC4806868.10.1371/journal.ppat.1005453

100. Martire S, Mosca L, d'Erme M: PARP-1 involvement in neurodegeneration: A focus on Alzheimer's and Parkinson's diseases. Mech Ageing Dev 2015, 146-148:536410.1016/j.mad.2015.04.001

101. Pieper AA, Verma A, Zhang J, Snyder SH: Poly (ADP-ribose) polymerase, nitric oxide and cell death. Trends Pharmacol Sci 1999, 20:171-181 


\section{Figures legends}

Figure 1. Multi-Dimensional Scaling (MDS) plot for the normalized expression data. Distance based the matrix of FPKM values quantified using RSEM v1.2.31 for all transcripts in all samples of macaques with detectable virus in the brain ( $n>3$ sequences) in red, and macaques with low/undetectable virus in the brain ( $n<3$ sequences) in blue. The plot shows good separation of the gene expression between the two groups, and non-overlapping.

Figure 2. Predicted significant activated or de-activated intracellular molecular pathways from frontal cortex with SIV infection. Active or non-active state of pathways were predicted using the IPA library of canonical pathways, and significance was based on z-score greater than 1.3. The figure shows the number of genes being differentially expressed per pathway. In orange or blue is indicated the activation score (z-score).

Figure 3. Differential expression of PARPs in the frontal cortex of macaques with detectable virus in the brain. (a) FPKMs (Fragments Per Kilobase of transcript per Million mapped reads) of PARP1, PARP9, PARP12 and PARP14 transcripts in macaques with detectable virus in the brain (red) and in macaques without detectable virus (blue). (b) Quantitative PCR analysis of mRNAs levels of PARP1, PARP9, PARP12 and PARP14 expressed in frontal cortex for macaques with detectable virus as relative to the averaged mRNA expression of the PARPs found in macaques without detectable virus. Colors indicate different macaques, while symbols are indicating the same macaque as shown in panel a. Asterisks indicate $p<0.001$. 
Figures

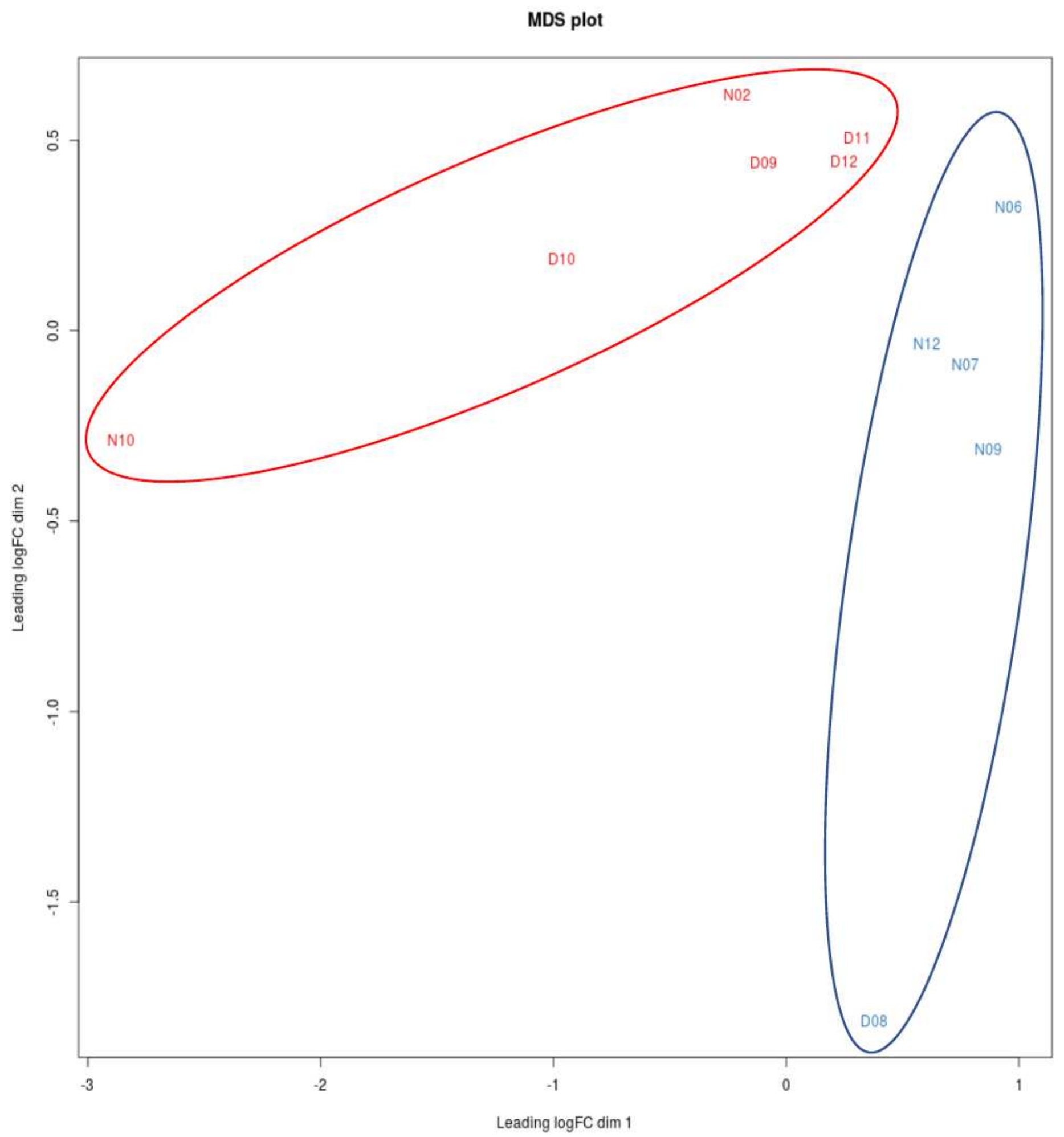

Figure 1

Multi-Dimensional Scaling (MDS) plot for the normalized expression data. Distance based the matrix of FPKM values quantified using RSEM v1.2.31 for all transcripts in all samples of macaques with detectable virus in the brain ( $n>3$ sequences) in red, and macaques with low/undetectable virus in the 
brain ( $\mathrm{n}<3$ sequences) in blue. The plot shows good separation of the gene expression between the two groups, and non-overlapping.

$$
-\log (p v a l u e)
$$

\section{Interferon signaling}

Role of pattern recognition of bacteria and viruses

Activation of IRF by cytosolic pattern recognition receptors

Neuroinflammation signaling pathway

Dendritic cell maturation

T-cell exhaustion signaling pathway

Complement system

Role of RIG1-like receptors in antiviral innate immunity

TREM1 signaling

Production of nitric oxide and reactive oxygen species in macrophages

Th1 pathway

Retinoic acid mediated apoptoris signaling

MIF-mediated glucocorticoid regulation

Toll-like receptor signaling

MIF regulation of innate immunity

Death receptor signaling

Phospholipases

Antioxidant action of vitamin C

Calcium-induced T lymphocyte apoptosis

Tec kinase signaling

iCOS-iCOSL signaling in T helper cells

Role of NFAT in regulation of immune response

Fcy receptor-mediated phagocytosis in macrophages and monocytes

PKC- $\theta$ signaling in T lymphocytes

NF-kB signaling

Colorectal cancer metastatis signaling

PI3K signaling in B lymphocytes

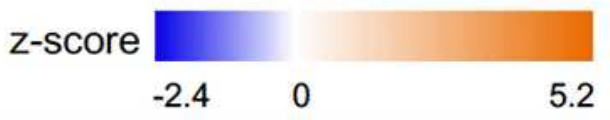

\section{Figure 2}

Predicted significant activated or de-activated intracellular molecular pathways from frontal cortex with SIV infection. Active or non-active state of pathways were predicted using the IPA library of canonical 
pathways, and significance was based on z-score greater than 1.3. The figure shows the number of genes being differentially expressed per pathway. In orange or blue is indicated the activation score (z-score).

a

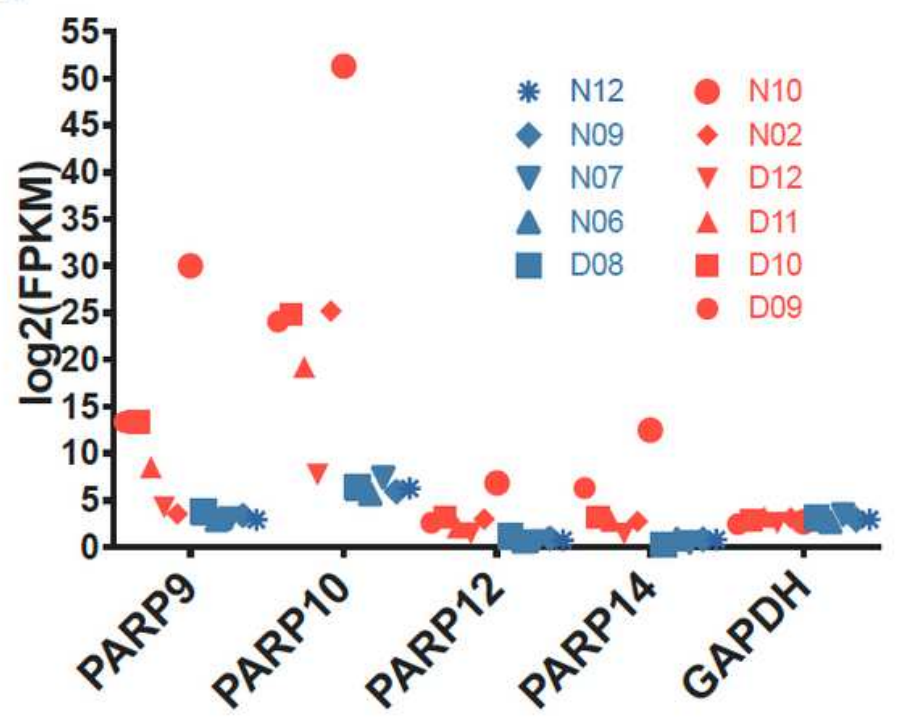

b

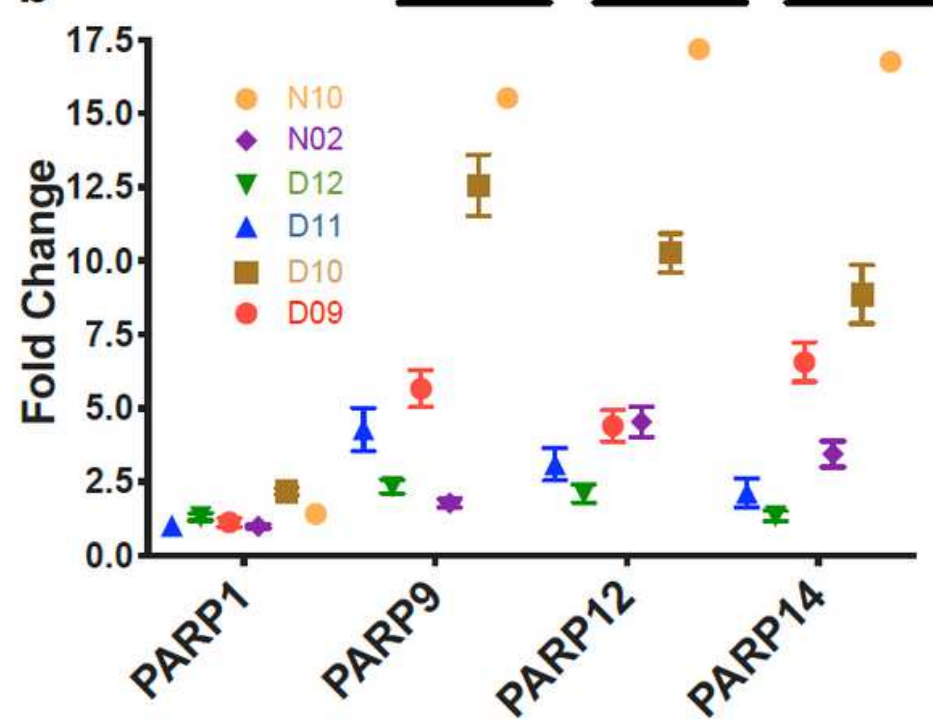

Figure 3

Differential expression of PARPs in the frontal cortex of macaques with detectable virus in the brain. (a) FPKMs (Fragments Per Kilobase of transcript per Million mapped reads) of PARP1, PARP9, PARP12 and PARP14 transcripts in macaques with detectable virus in the brain (red) and in macaques without detectable virus (blue). (b) Quantitative PCR analysis of mRNAs levels of PARP1, PARP9, PARP12 and PARP14 expressed in frontal cortex for macaques with detectable virus as relative to the averaged mRNA expression of the PARPs found in macaques without detectable virus. Colors indicate different macaques, while symbols are indicating the same macaque as shown in panel a. Asterisks indicate $\mathrm{p}<$ 0.001 .

\section{Supplementary Files}

This is a list of supplementary files associated with this preprint. Click to download.

- Supplementarymaterials.docx

- TableS3.docx

- Tables5.xls

- Tables7.xls 